\title{
The influence of differences in neurocognitive function on lower limb kinematics, kinetics, and muscle activity during an unanticipated cutting motion
}

\author{
Satoshi SHIBATA, MS, $\mathrm{PT}^{1}$, \\ Masahiro TAKEMURA, Master of Physiotherapy(Sports), $\mathrm{PT}^{2}$ and Shumpei MiYAKAWA, PhD, MD² \\ ${ }^{1)}$ Department of Sports Medicine Graduate School of Comprehensive Human Sciences, University of Tsukuba \\ ${ }^{2)}$ Faculty of Health and Sport Sciences, University of Tsukuba
}

\begin{abstract}
Background: Neurocognitive function may be a risk factor for anterior cruciate ligament (ACL) injury by changing neuromuscular control such as muscle activities. However, the effect of differences in neurocognitive function on biomechanics and neuromuscular control related to ACL injury risk is not clear. The purpose of this study was to examine the effect of differences in neurocognitive function on biomechanics and neuromuscular control during an unanticipated side-step cutting motion. Method: The subjects were 15 collegiate female athletes who were divided into two groups using the Symbol Digit Modalities Test (SDMT). The experimental task was an unanticipated side-step cutting motion from a $30 \mathrm{~cm}$ high box. We calculated joint angles and moments using a 3-dimensional motion analysis system from the dominant leg, and measured muscle activities using a surface electromyography. We calculated the co-contraction ratio (CCR) as relative muscle activity of the quadriceps to the hamstring. Results: As a result, subjects with a lower SDMT score had significantly increased quadriceps activity before and after ground contact and decreased CCR only after ground contact. Conclusion: In the lower SDMT score group, the quadriceps showed stronger muscle activity than the hamstring during an unanticipated side-step cutting motion. This dominant quadriceps muscle activity has been reported to increase the load on the ACL, and there was a possibility of increasing the risk of the ACL injury. Considering these factors, subjects with lower neurocognitive function could have a high risk of ACL injury due to alterations in muscle activities surrounding the knee.
\end{abstract}

Key words: ACL injury, motion analysis, neurocognitive function, unanticipated cutting motion

(Phys Ther Res 21: 44-52, 2018)

$\mathbf{P}$ have been studied as risk factors related to anterior cruciate ligament (ACL) injury ${ }^{1-3)}$. Poor neurocognitive function was pointed out as a new risk factor for ACL injury recently ${ }^{4,5)}$. Swanik et al. ${ }^{4)}$ measured the neurocognitive function using "ImPACT", which was mainly used as a neurocognitive function test for concussion and scored by a computer-

Received: November 6, 2017

Accepted: July 2, 2018

Advance Publication by J-STAGE: August 30, 2018

Correspondence to: Satoshi Shibata, Department of Sports Medicinem Fraduate School of Comprehensive Human Sciences, University of Tsukuba, 1-1-1 Tennodai, Tsukuba, Ibaraki 305-8574, Japan \# e-mail: satoshibata96@gmail.com

doi: 10.1298/ptr.E9938 based method. They then compared mean scores of the two groups: a non-contact ACL injured group and a control group $^{4}$. They reported that as for reaction time, processing speed, and visual/verbal memory, the non-contact ACL injured group showed low scores, and they suggested that poor neurocognitive function may be a risk factor of ACL injury ${ }^{4)}$. In addition, a prospective cohort study had examined the relationship between the reaction time in the "ImPACT" and the subsequent occurrence of lower limb sprain and strain among college football players ${ }^{5)}$. It was found that as the reaction time extended, the incidence of a lower limb injury increased, and the cut-off value was set to 0.545 $\mathrm{sec}^{5)}$. The sensitivity of this cut-off value was $74 \%$, the specificity was $51 \%$, and the odds-ratio was $2.94^{5)}$. Furthermore, prolonged neurocognitive reaction time was shown 
Table 1. HP and LP group characteristics (mean $\pm \mathrm{SD})$.

\begin{tabular}{lrcc}
\hline & HP group $(\mathrm{n}=7)$ & LP group $(\mathrm{n}=8)$ & $P$ value \\
\hline SDMT achievement rate $(\%)$ & $70.8 \pm 5.0$ & $60.0 \pm 3.1$ & $<0.001 *$ \\
\hline Age (years) & $20.0 \pm 1.4$ & $20.3 \pm 1.3$ & 0.930 \\
Body height $(\mathrm{cm})$ & $166.8 \pm 8.4$ & $166.4 \pm 6.7$ & 0.912 \\
Body weight $(\mathrm{kg})$ & $60.8 \pm 8.2$ & $60.5 \pm 6.0$ & 0.725 \\
\hline Q-angle $\left(^{\circ}\right)$ & $10.0 \pm 2.0$ & $10.1 \pm 1.1$ & 0.887 \\
Thigh-foot angle $\left({ }^{\circ}\right)$ & $7.7 \pm 9.1$ & $4.0 \pm 8.7$ & 0.435 \\
Navicular drop test $(\mathrm{cm})$ & $4.1 \pm 0.7$ & $3.8 \pm 0.4$ & 0.331 \\
General joint laxity & $2.2 \pm 1.4$ & $2.4 \pm 0.8$ & 0.789 \\
\hline
\end{tabular}

*There was a significant difference between HP and LP $(\mathrm{p}<0.05)$.

to be a predictor of lower limb sprain and strain. These reports indicated that poor neurocognitive function is related to the occurrence of injury, especially ACL injury. However, it is not clear how poor neurocognitive function causes the injury.

It has been reported that the biomechanical risk factors of ACL injury were decreased knee flexion angle, increased knee valgus angle, increased knee abduction moment, and increased ground reaction force $(\mathrm{GRF})^{1-3,6)}$. In addition, alterations in muscle activities during motion were reported as neuromuscular control factors of ACL injuries ${ }^{1-3,7-12)}$. The influence of the imbalance of muscle activities of the quadriceps and hamstring has been studied, especially the strong contraction of the quadriceps has been reported to increase the risk of the ACL injury ${ }^{10-12}$.

Neurocognitive function is composed of visual attention, self-monitoring, agility/fine motor performance, processing speed/reaction time, and dual-tasking ${ }^{13)}$. Olsen et al. ${ }^{14)}$ found from video analysis that an athlete's attention at the time of an ACL injury was towards opponents and goals. Therefore, we consider that an athlete with the poor neurocognitive function might perform susceptible motions in the following situations: the next play selection, sudden change of the landing point, or unexpected motion. Therefore, an athlete with poor neurocognitive function may have a high risk of ACL injury due to fatal biomechanical errors and poor neuromuscular control during sports activity. However, the influence of the neurocognitive function on complex tasks is not clear. Herman et al. ${ }^{15)}$ studied the relationship between neurocognitive function using a computerbased test called the Concussion Resolution Index (CRI) and kinematics and kinetics of lower limbs during an unanticipated landing motion. They grouped the subjects into a higher-performers (HP) group and a lower-performers (LP) group according to the CRI score ${ }^{15)}$. The LP group showed a significant increase in peak vertical ground-reaction force, an increased peak anterior tibial shear force, increased knee adduction moment, and increased knee valgus angle, as well as a decreased trunk flexion angle ${ }^{15)}$. These results showed that if an athlete had poor neurocognitive function, there was a high-risk of ACL injury ${ }^{15)}$. However, the relationship between muscle activity and neurocognitive function during unanticipated motion has not been studied.

The purpose of this study was to clarify the difference of an unanticipated side-step cutting motion between a higher performance (HP) group and a lower performance (LP) group in a neurocognitive function test. We hypothesized that the LP group would demonstrate a lesser knee flexion angle, greater knee valgus angle, greater hip adduction angle, and greater hip internal rotation angle. In kinetics, a greater knee valgus moment, greater hip adduction moment, and a greater vertical GRF could be generated. As for muscle activities, the co-contraction ratio (CCR) of the quadriceps and the hamstring would increase, as a consequence of increased quadriceps and decreased hamstring muscle activities.

\section{Method}

\section{Participants}

Subjects were 15 female athletes (mean \pm SD age: $20.1 \pm 1.3$ years; height $1.67 \pm 0.7 \mathrm{~m}$; weight: $60.6 \pm 6.9$ $\mathrm{kg}$ ), playing sports including jumping/cutting motions (e.g., basketball and soccer) in university athletic clubs. They belonged to the highest national competition level, and they were practicing 2-3 hours a day, 5-6 days a week. Exclusion criteria were any injuries in the lower limbs, a concussion within the past six months, any disorder of the peripheral sensory system, or a past history of surgery in the lumber spine or lower limbs. We collected the data from the following measurements from their dominant leg to remove any anatomical characteristics that could be a risk factor of ACL injury: Q-angle, thigh-foot angle, navicular drop test, and general joint laxity ${ }^{16)}$ (Table 1). All subjects provided written informed consent before their participation. This study was approved by the Ethical Committee of the Faculty of Health and Sports Sciences at the University of Tsukuba (approval number. 28-37). 


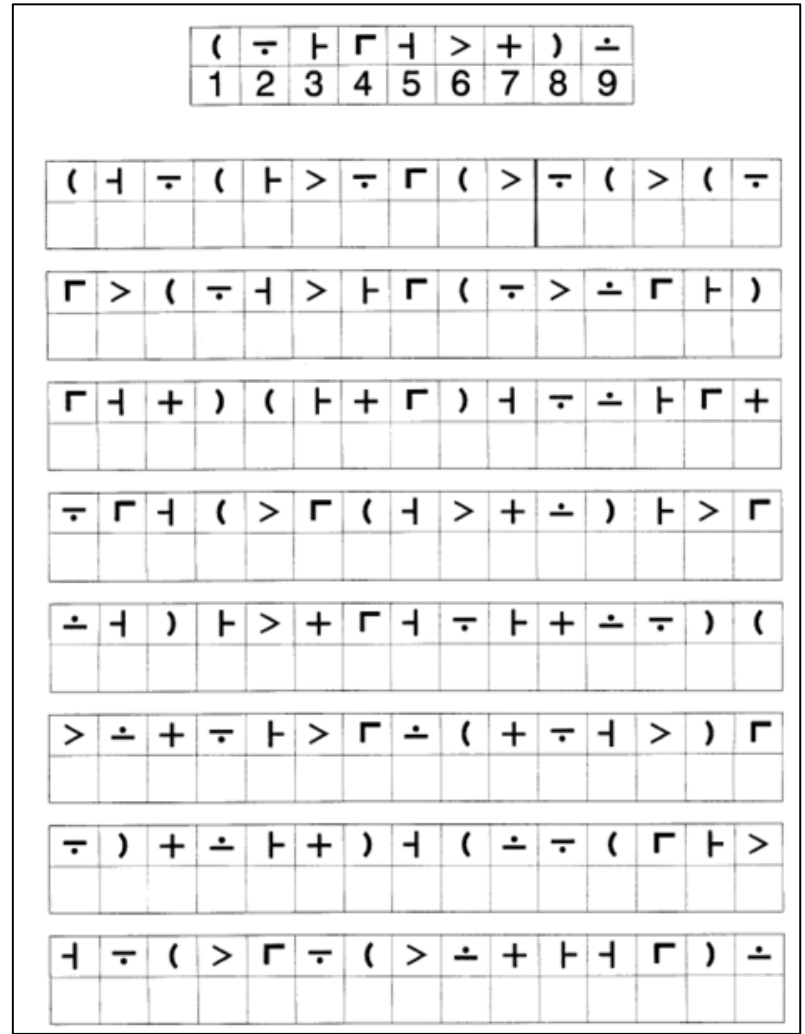

Figure 1. The symbol digit modalities test (SDMT).

\section{Neurocognitive Test}

Neurocognitive testing was performed using the Symbol Digit Modalities Test (SDMT) ${ }^{17)}$. Participants were asked to fill out 110 boxes under symbols with the corresponding number within 90 seconds, referring to a key on top of the test form to identify which number goes with each symbol ${ }^{17,18)}$ (Fig. 1). The number of correct answers were counted, and the achievement rate $(\%)$ was calculated for analysis and grouping the subjects, by dividing the number of the correct answers by 110 . The subjects were grouped into a HP group and a LP group according to the achievement rate. The median of SDMT was used as a cutoff value to group the subjects, since a standard cut-off value of the SDMT has not been defined ${ }^{18)}$. It was reported that SDMT is a test to evaluate psychomotor speed, visual short-term memory, attention, and concentration ${ }^{19)}$. Reliability of SDMT is reported to be $0.82-0.84^{19)}$. SDMT is mainly used for functional evaluation before and after concussion ${ }^{20)}$ and evaluation of the neurocognitive function for multiple sclerosis $^{21,22}$. The validity of SDMT was examined with a paper-and-pencil test as well as a computer-based test ${ }^{18)}$. SDMT was measured at the same time as the motion analysis was done.

\section{Experimental Tasks (the unanticipated cutting task)}

Three motions were selected as experimental tasks: side-step cutting 45 degrees (CUT), single-leg landing (LAND), and forward stepping (STEP) using the dominant leg. The dominant leg was defined as the leg with which the subjects would prefer to kick a ball. The subjects wore spandex athletic tops and shorts and were barefoot, and jumped off a center of force platform from a $30 \mathrm{~cm}$ high box placed at its edge. They were then asked to perform one of the tasks according to a command on a personal computer (PC) monitor immediately after leaving the box. The PC was synchronized with a foot switch at the top of the box and displayed one of the tasks at random. Therefore, they were forced to alter their motion before landing on the force platform with unanticipated timing ${ }^{23,24}$. The PC monitor was set $4 \mathrm{~m}$ away from the box at a height of 30 cm (Fig. 2). Instructions for each experimental task were given on the monitor: (1) CUT: when a yellow horizontal arrow is displayed, (2) LAND: when a blue circle is displayed, and (3) STEP: when a red upward arrow is displayed. We showed the direction of motion with tape on the ground and subjects were instructed to step to one of the directions (Fig. 3). In this research, we analyzed only the task of CUT.

\section{Data Collection (kinematic, kinetic and EMG)}

A three-dimensional motion analysis system, the VICON MX motion analysis system (VOCON, Oxford, UK) was used to capture the task motions with a $250 \mathrm{~Hz}$ sampling rate through 10 infrared cameras. Thirty-five retroreflective markers were placed over the whole body of each subject in a standard Plug-in Gait model (Helen Hays marker-set) on anatomical landmarks ${ }^{25,26)}$.

Ground reaction force data was obtained at $1,000 \mathrm{~Hz}$ from a force platform (Kistler Instruments, Inc., model 9281C, Winterthur, Switzerland) which was synchronized with the kinematic data. Joint moments were calculated on the dominant leg side through a full inverse-dynamic model implemented by the use of VICON Plug-in Gait. The estimated joint moments were normalized by the body weight of the subject. Joint moments were reported as external torques.

Surface electromyography (EMG) data were recorded at 1,500 Hz from a 7-channel EMG system (Telemyo DTS, Noraxon Inc.; Scottsdale, AZ, USA) and collected synchronously with motion and force platform data. Bipolar surface electrodes $(\mathrm{Ag}-\mathrm{AgCl})$ were separated by $2 \mathrm{~cm}$ and placed on the following seven muscles: gluteus medius (GMed), adductor longus (Add), rectus femoris (RF), vastus medialis (VM), vastus lateralis (VL), biceps femoris (BF), and semimembranosus (SM). Each electrode was placed as follows: GMed, approximately $2 \mathrm{~cm}$ below of the midpoint of the iliac crest over the muscle belly; Add, approximately 10 $\mathrm{cm}$ below the pubic symphysis over the muscle belly; RF, approximately halfway between the patellar upper part and the anterior superior iliac spine over the muscle belly; VM, approximately $5 \mathrm{~cm}$ from the medial patellar at 45 degrees over the muscle belly; VL, approximately distal two thirds 
A

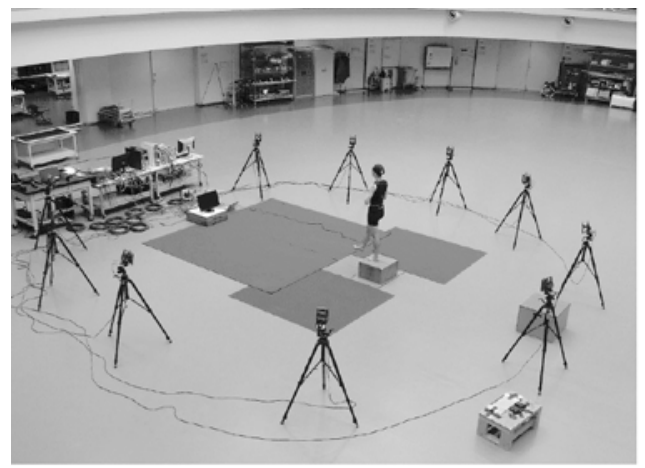

B
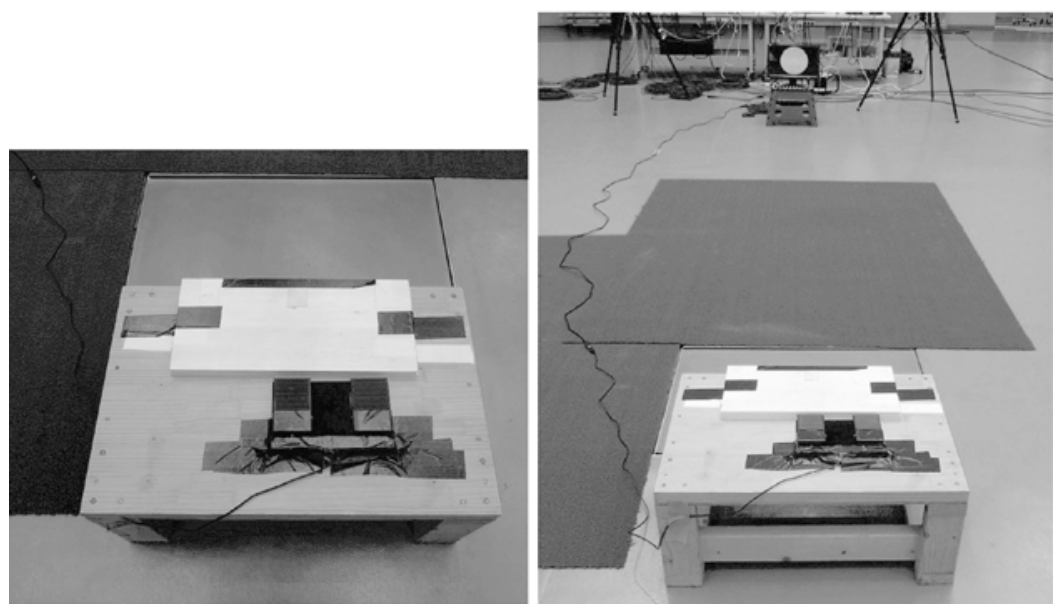

Figure 2. A: Experimental environment.

B: Footswitch and $30 \mathrm{~cm}$ high box.

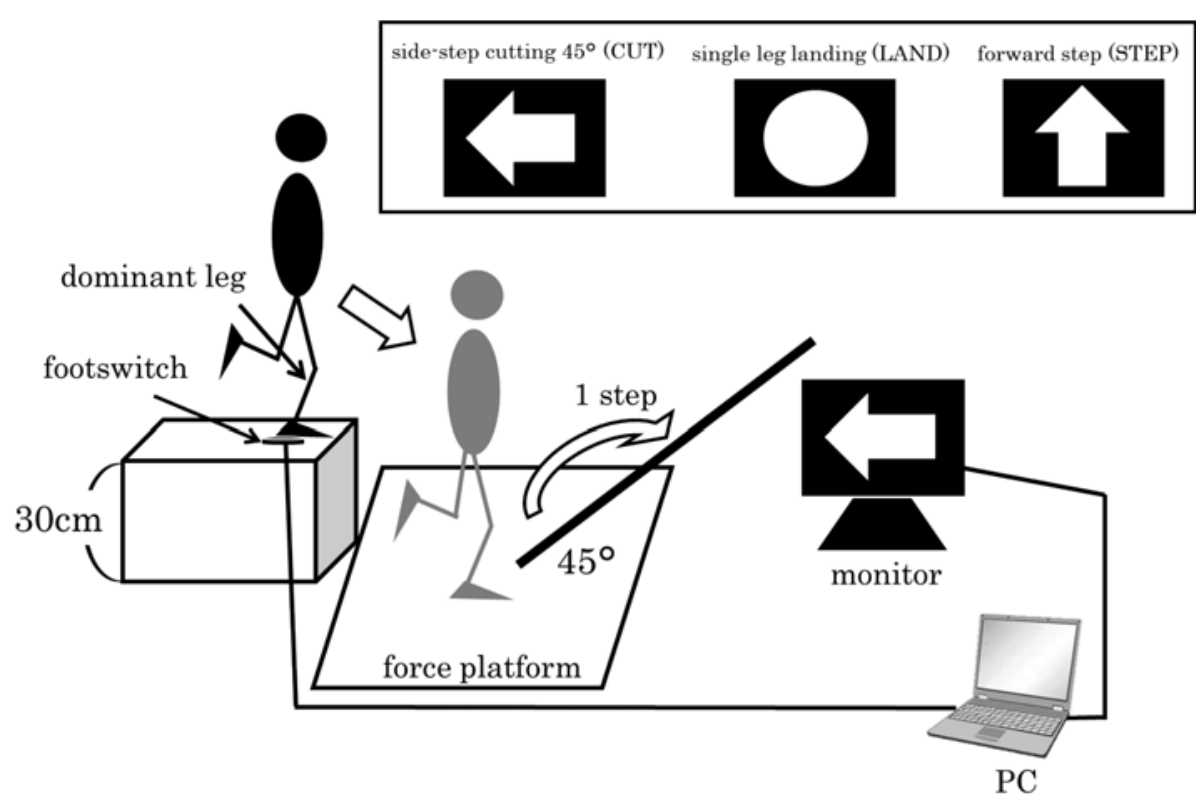

Figure 3. The procedure of unanticipated side-step cutting task.

(1) Subject stood by stepping on the footswitch with their dominant leg on a $30 \mathrm{~cm}$ high box.

(2) Immediately after the subject jumped off the box, an experimental task was displayed on the monitor.

(3) Subject reacted to the instruction displayed on the monitor.

(4) Side-step cutting motion landed on the force platform with the dominant leg and immediately took one step in the direction of 45 -degrees. 
Table 2. Peak kinematics and kinetics variables comparing HP and LP groups performing an unanticipated cutting motion (mean \pm SD).

\begin{tabular}{lcccc}
\hline & & HP group & LP group & $P$ value \\
\hline Hip angle $\left(^{\circ}\right)$ & Flexion & $37.0 \pm 5.0$ & $36.6 \pm 10.3$ & 0.929 \\
& Adduction & $4.7 \pm 3.7$ & $0.0 \pm 5.7$ & 0.087 \\
& Internal rotation & $-2.1 \pm 4.8$ & $-3.6 \pm 10.5$ & 0.730 \\
Knee angle $\left(^{\circ}\right)$ & Flexion & $62.0 \pm 5.3$ & $55.4 \pm 8.8$ & 0.111 \\
& Valgus & $14.6 \pm 10.1$ & $10.7 \pm 5.7$ & 0.382 \\
GRF $(\% \mathrm{BW})$ & Vertical & $5.5 \pm 1.0$ & $5.3 \pm 1.0$ & 0.621 \\
Hip moment & Flexion & $33.5 \pm 16.5$ & $26.8 \pm 25.8$ & 0.568 \\
$(\mathrm{Nm} / \mathrm{kg})$ & Adduction & $22.8 \pm 8.9$ & $21.9 \pm 8.3$ & 0.851 \\
$\mathrm{Knee} \mathrm{moment}$ & Flexion & $53.5 \pm 11.1$ & $54.0 \pm 6.5$ & 0.927 \\
$(\mathrm{Nm} / \mathrm{kg})$ & Abduction & $5.6 \pm 3.2$ & $8.5 \pm 4.2$ & 0.165 \\
\hline
\end{tabular}

*There was a significant difference between HP and LP $(\mathrm{p}<0.05)$.

between the patellar lateral side and the anterior superior iliac spine over the muscle belly; SM and BF, approximately halfway between the tibial lateral epicondyle and the ischial tuberosity over the muscle belly. The skin was shaved and exfoliated using a skin treatment agent then cleaned with an alcohol swab before electrode placement.

The subjects conducted several practical trials and experienced a comfortable trial before starting data collection. Each subject completed three trials for each motion pattern. After completion of all nine trials, the subjects' participation in the study ended.

\section{Data Analysis}

The raw kinematic and ground reaction force data were filtered based on a frequency content analysis of the digitized coordinate data. Marker trajectories were filtered at $7 \mathrm{~Hz}$ using a 4th-order Butterworth filter using VICON Nexus 1.6.1 software (Oxford Metrics Ltd., UK). The kinematics and kinetics data normalized the stance phase to 101 points. As described in a previous paper ${ }^{27)}$, 'initial contact' (IC) was defined as the time where vertical ground reaction force is higher than $10 \mathrm{~N}$, and 'weight acceptance' (WA) was defined as the first $30 \%$ of stance. For all kinematics and kinetics data, the peak value in the WA phase were calculated.

For EMG data, the stored raw signals were band-pass filtered $(20-500 \mathrm{~Hz})$, and root mean square (RMS) processed with a $10 \mathrm{~ms}$ time constant using Myomuscle (Noraxon Inc.; Scottsdale, AZ, USA). Before data collection, the RMS data were then normalized by the maximum voluntary contraction (MVC) for each muscle (\% MVC). The MVC was recorded for 5-seconds, and the average amplitude determined from the stable RMS for 3-seconds.

The averaged activity of the knee flexors (HAM) were calculated from BF and SM, and the knee extensors (QUAD) were calculated from VM and VL. We calculated $\mathrm{CCR}$ (co-contraction ratio) as the relative muscle activity of the QUAD to the $\mathrm{HAM}^{28,29}$. High CCR indicated greater
HAM activity relative to QUAD activity. Low CCR indicated less HAM activity relative to QUAD activity.

EMG data were recorded for $50 \mathrm{~ms}$ before IC (pre-IC) and the first $50 \mathrm{~ms}$ (post-IC) of the cutting phase, as in the previous research ${ }^{30}$. This was done for the following reasons: (1) we chose $50 \mathrm{~ms}$ before IC because this is appropriate for individual pre-planned muscle recruitment strategy $^{31)}$, and (2) we chose the first $50 \mathrm{~ms}$ of the stance phase for side-step cutting to assess muscle activation immediately after IC because ACL injuries occur within approximately $50 \mathrm{~ms}$ after $\mathrm{IC}^{32-34)}$.

\section{Statistical Analysis}

Results were presented as mean \pm standard deviation (SD). We used an unpaired t-test to compare the differences between the LP and the HP groups. Statistical significance was set at $\mathrm{p}<0.05$. All statistical analyses were performed using SPSS statistics 22.0 (IBM, SPSS Tokyo, Japan).

\section{Results}

For all subjects, the average SDMT achievement rate was $65.0 \pm 6.8 \%$ and the median was $63.6 \%$. From this result, the top 7 subjects were allocated to the HP group and the remaining eight subjects were allocated to the LP group. The achievement rate in the HP was significantly larger than that of the LP (HP: $70.8 \pm 5.0 \%$, LP: $60.0 \pm 3.1 \%$; $<<$ 0.001) (Table 1).

There were no significant differences between the two groups in the peak joint angles and moments (Table 2). There were no significant differences in the HAM activity between the groups. The QUAD activity in the LP was significantly larger than that of the HP at pre-IC (HP: $40.7 \pm$ 21.5\%, LP: $78.4 \pm 37.9 \% ; \mathrm{p}=0.038)$. The LP was significantly larger than HP at post-IC (HP: $80.8 \pm 26.7 \%$, LP: $137.2 \pm 54.0 \% ; \mathrm{p}=0.027)$. The CCR of HP was larger than those of LP at pre-IC (HP: $158.3 \pm 150.5 \%$, LP: $59.1 \pm$ $21.7 \%$ ), and HP was significantly larger than LP at post-IC 
Table 3. Muscle activities variables comparing HP and LP groups before and after the IC (mean $\pm \mathrm{SD})$.

\begin{tabular}{lccrl}
\hline & & HP group & LP group & $P$ value \\
\hline HAM $(\%$ MVC) & pre-IC & $42.7 \pm 14.6$ & $41.4 \pm 12.2$ & 0.853 \\
& post-IC & $36.4 \pm 16.0$ & $37.0 \pm 17.5$ & 0.946 \\
QUAD (\%MVC) & pre-IC & $40.7 \pm 21.5$ & $78.4 \pm 37.9$ & $0.038 *$ \\
& post-IC & $80.8 \pm 26.7$ & $137.2 \pm 54.0$ & $0.027 *$ \\
CCR $(\%)$ & pre-IC & $158.3 \pm 150.5$ & $59.1 \pm 21.7$ & 0.087 \\
& post-IC & $50.8 \pm 27.1$ & $28.0 \pm 10.7$ & $0.046 *$ \\
\hline
\end{tabular}

HAM was the average muscle activity of the knee flexor and calculated from BF and SM. QUAD was the average muscle activity of the knee extensor and calculated from VM and VL. The co-contraction ratio (CCR) was calculated as a relative muscle activity of the QUAD to the HAM.

$*$ There was a significant difference between HP and LP $(\mathrm{p}<0.05)$.

(HP: $50.8 \pm 27.1 \%$, LP: $28.0 \pm 10.7 \%$; $\mathrm{p}=0.046)$ (Table 3).

\section{Discussion}

We investigated the influence of differences in neurocognitive function measured by SDMT on lower limb kinematics, kinetics, and muscle activities during an unanticipated side-step cutting motion. In this study, there were no significant differences between the two groups in the lower limb kinematics and kinetics. In the LP group, increased QUAD activities at the pre-IC and post-IC, and decreased CCR at post-IC were recorded. These results supported our hypothesis. To our knowledge, this study is the first study showing an association of neurocognitive function with muscle activities, using a paper-and-pencil neurocognitive function test called SDMT.

Many previous studies had pointed out that an imbalance of the muscle activities between the quadriceps and hamstring strongly influences the occurrence of an ACL injury $^{7-10)}$. The knee slightly flexes immediately after the IC, and the tibia is pulled out anteriorly against the femur by a quadriceps contraction. This traction force acts as an anterior shear force on the tibia. Therefore, the quadriceps dominant muscle activity at the time of landing increases the risk of ACL injury by increasing the load on the $\mathrm{ACL}^{11,12)}$. In addition, the hamstring protectively acts as an inhibitor of shear force, working as an antagonist ${ }^{12,35)}$. Thus, strongly contracting the quadriceps muscle immediately after ground contact could hurt the knees ${ }^{36}$. In the LP group of this study, muscle activity dominated by the quadriceps occurred soon after IC, and we could say that it is a muscle activity with a high risk of ACL injury.

It was reported that the normative data of SDMT was $58.2 \pm 9.1$ (converting to an achievement rate: approximately $53 \%$ ) as the number of correct answers for young adults $(<30 \text { years })^{37}$. For all subjects of this current study, the average of the SDMT achievement rate was $65.0 \pm$ $6.8 \%$. The average score of the LP group was $60.0 \pm 3.1 \%$, while the lowest value of the subject was $55.5 \%$. Therefore, it was considered that the score of the subjects of this study was higher than the normative data. Thus, since the subjects of this current study have good neurocognitive function measured by SDMT, it was considered that no difference was observed between groups in kinematics and kinetics during an unanticipated cutting motion.

The relationship between lower limb kinematics and kinetics during an unanticipated landing motion and neurocognitive function had been studied using a computer test called the Concussion Resolution Index $(\mathrm{CRI})^{15}$. In a previous study ${ }^{15}$, the lower CRI score group showed a significant increased in peak vertical ground-reaction force, increased peak anterior tibial shear force, increased knee abduction moment, and an increased knee abduction angle, as well as a decreased trunk flexion angle ${ }^{15)}$. These results showed that athletes with a lower baseline neurocognitive function generated knee kinematics and kinetics that increased the ACL injury risk ${ }^{15}$. The results from this previous research ${ }^{15)}$ were different from our study, in which the influence was not clear for kinematics and kinetics due to differences in neurocognitive function.

The reason why different results were obtained in our current research compared to the previous research ${ }^{15)}$ might be that the experimental methods and the selection criteria of the subjects were different. First, in the current study, the subjects were only female athletes in a high-level of competition, whereas the previous study ${ }^{15)}$ included both male and female recreational level athletes. However, female athletes are known to have a higher incidence of ACL injury than male athletes ${ }^{38,39)}$ and the causes are diverse ${ }^{1-3)}$. Especially, it has been reported that there is a sex difference between biomechanics and neuromuscular control in landing and cutting motion ${ }^{34,40)}$. Therefore, in a biomechanics study such as the current study, the sex of the subjects might influence the results. The competition level dose not influence the ACL injury rate ${ }^{41,42)}$. However, it was reported that there are differences in the biomechanics of the knee de- 
pending on the competition level during an unanticipated cutting task $^{24,43}$. For recreational level athletes, but not high competition level athletes, there is a significant effect on the frontal plane knee kinematics and kinetics during a single-leg land-and-cut motion ${ }^{24}$.

Second, for the neurocognitive function test, the current study used a paper-and-pencil test, whereas the previous study ${ }^{15)}$ used a computer test. SDMT only examined factors such as psychomotor speed, visual short-term memory, attention, and concentration in neurocognitive functions ${ }^{18,199}$. As components of neurocognitive function, visual attention, self-monitoring, agility/fine motor performance, processing speed/reaction time, and dual-tasking have been reported $^{13)}$. Therefore, it was not possible to evaluate all components of neurocognitive function with SDMT alone. From the above, the current study examined the influence of part of the neurocognitive function and unanticipated side-step cutting motion.

Third, experimental tasks also had unanticipated settings: they differed between the side-step cutting and the single-leg landing ${ }^{15}$, which was thought to affect the results. Thus, there are some differences in the methods of this research and previous research ${ }^{15}$. There has been no other prior study that has examined the relationship between neurocognitive function and biomechanics. Therefore, further study is necessary for the interpretation of the results.

It was reported that neurocognitive function examined by SDMT had the four following components: psychomotor speed, visual short-term memory, attention (especially ability to divide attention), and concentration ${ }^{19}$. The decreased SDMT score could lead to poor neuromuscular control, especially psychomotor speed and attention components could be related. For example, an athlete with fine psychomotor speed and attention may rapidly adjust to new demands during sports activities while maximizing sports performance and safety. Conversely, low psychomotor speed may reduce the ability to keep reaction to variable demands during sports activities within an adequate time to allow for safe and appropriate responses. Furthermore, when the ability to divide attention is insufficient, it would be difficult to pay visual attention to the surrounding situation and to monitor the neuromuscular performance associated with the athletic task appropriately at the same time. Thus, an athlete with a low SDMT score may have poor biomechanics and neuromuscular control ${ }^{13)}$.

Herman et al. ${ }^{13)}$ described the association of impairment of neurocognitive function with increased musculoskeletal injury risk. It has been reported that poor neurocognitive function causes a decrease in the visual attention, self-monitoring, agility and fine motor performance, processing speed and reaction time, and dual tasking ${ }^{13}$. Factors that cause impairment of neurocognitive function were inadequate sleep, concussion, psychological stress, and poor baseline neurocognition ${ }^{13)}$. By understanding and control- ling these factors, neurocognitive functions can be preserved, and it may lead to the prevention of injuries.

From the results of this current study, it was suggested that a lower SDMT score leads to poor neuromuscular control that the muscle activity of the quadriceps dominates during unanticipated side-step cutting motions. It has been reported that poor neuromuscular control was an important risk factor for ACL injury ${ }^{1-3)}$. Therefore, we considered that lower neurocognitive function was a part of the increased risk factors of ACL injury by causing poor neuromuscular control. However, there are still many problems when using neurocognitive function as screening for the risk factors for ACL injury. We do not know which test is appropriate, such as a paper-and-pencil test like SDMT or a computer test like ImPACT. Furthermore, the cut-off value of a neurocognitive function test is unknown. Therefore, when using a neurocognitive function test for ACL injury risk screening, there are still many problems and further study is necessary.

There were several limitations in this current study. First, we used a neurocognitive function test based on a conventional paper-and-pencil test, while computer tests are now widely used. Paper-and-pencil tests have been able to investigate only a single component. In addition, paperand-pencil tests have lower reliability compared with computer tests ${ }^{18,44}$. However, measuring neurocognitive function using a computer for athletes is difficult in Japan due to high cost and lower recognition.

Second, although the visual stimulus we used in the current study was to display a symbol such as a simple arrow on a monitor, the stimulus condition does not necessarily match the situation of an actual game and may be a little too simple. Our research was performed in a laboratory setting, but a cutting motion would be different in a true sports situation. Therefore, in future research, it is necessary to examine a setting close to actual game conditions.

Third, we did not measure lower limb muscle strength in this study. It was not clear whether the difference in muscle strength could be a risk factor for ACL injury ${ }^{1-3)}$. Moreover, there was no consensus on the effect of lower limb strength on side-step cutting motion ${ }^{27,29}$, but differences in muscle strength might cause a difference in muscle activity. It may be necessary to examine the influence of the difference in muscle strength on motion.

\section{Conclusion}

In this current study, we examined the difference in lower limb kinematics, kinetics, and muscle activities during an unanticipated side-step cutting motion due to the difference in neurocognitive function. As a result, there were no significant differences in the lower limb kinematics and kinetics, while the QUAD activity of the LP was increased pre-IC and post-IC, and was decreased in the CCR at post- 
IC. In other words, subjects with lower neurocognitive function were observed to have muscle activity patterns dominating the quadriceps. These altered muscle activities could increase the risk of ACL injury ${ }^{11,12}$.

Conflict of Interest: We have no financial conflicts of interest to disclose.

\section{References}

1) Griffin LY, Albohm MJ, et al.: Understanding and preventing noncontact anterior cruciate ligament injuries: a review of the Hunt Valley II meeting, January 2005. Am J Sports Med. 2006; 34: $1512-1532$.

2) Griffin LY, Agel J, et al.: Noncontact anterior cruciate ligament injuries: risk factors and prevention strategies. J Am Acad Orthop Surg. 2000; 8: 141-150.

3) Hewett TE, Myer GD, et al.: Anterior cruciate ligament injuries in female athletes: part 1, mechanisms and risk factors. Am J Sports Med. 2006; 34: 299-311.

4) Swanik CB, Covassin T, et al.: The relationship between neurocognitive function and noncontact anterior cruciate ligament injuries. Am J Sports Med. 2007; 35: 943-948.

5) Wilkerson GB: Neurocognitive reaction time predicts lower extremity sprains and strains. Int J Athl Ther Train. 2012; 17: 4-9.

6) Hewett TE, Myer GD, et al.: Biomechanical measures of neuromuscular control and valgus loading of the knee predict anterior cruciate ligament injury risk in female athletes: a prospective study. Am J Sports Med. 2005; 33: 492-501.

7) Renström $P$, Arms SW, et al.: Strain within the anterior cruciate ligament during hamstring and quadriceps activity. Am J Sports Med. 1986; 14: 83-87.

8) Torzilli PA, Deng X, et al.: The effect of joint-compressive load and quadriceps muscle force on knee motion in the intact and anterior cruciate ligament-sectioned knee. Am J Sports Med. 1994; 22: 105-112.

9) Novak PJ, Bach BR, et al.: Cost containment: a charge comparison of anterior cruciate ligament reconstruction. Arthroscopy. 1996; 12: 160-164.

10) Beynnon BD and Fleming BC: Anterior cruciate ligament strain in-vivo: a review of previous work. J Biomech. 1998; 31: 519525 .

11) Markolf KL, Gorek JF, et al.: Direct measurement of resultant forces in the anterior cruciate ligament: an in vitro study performed with a new experimental technique. J Bone Joint Surg Am. 1990; 72: 557-567.

12) Solomonow M, Baratta R, et al.: The synergistic action of the anterior cruciate ligament and thigh muscles in maintaining joint stability. Am J Sports Med. 1987; 15: 207-213.

13) C Herman D, Zaremski JL, et al.: Effect of neurocognition and concussion on musculoskeletal injury risk. Curr Sports Med Rep. 2015; 14: 194-199.

14) Olsen OE, Myklebust G, et al.: Injury mechanisms for anterior cruciate ligament injuries in team handball: a systematic video analysis. Am J Sports Med. 2004; 32: 1002-1012.
15) Herman DC and Barth JT: Drop-jump landing varies with baseline neurocognition: implications for anterior cruciate ligament injury risk and prevention. Am J Sports Med. 2016; 44: 23472353.

16) Bonci CM: Assessment and evaluation of predisposing factors to anterior cruciate ligament injury. J Athl Train. 1999; 34: 155164.

17) Smith A: Symbol Digit Modalities Test. Western Psychological Services, Los Angels, CA, 1982.

18) Randolph $\mathrm{C}$, McCrea $\mathrm{M}$, et al.: Is neuropsychological testing useful in the management of sport-related concussion? J Athl Train. 2005; 40: 139-152.

19) Register-Mihalik JK, Kontos DL, et al.: Age-related differences and reliability on computerized and paper-and-pencil neurocognitive assessment batteries. J Athl Train. 2012; 47: 297-305.

20) Hinton-Bayre AD, Geffen GM, et al:: Concussion in contact sports: reliable change indices of impairment and recovery. J Clin Exp Neuropsychol. 1999; 21: 70-86.

21) Benedict RHB, Smerbeck A, et al.: Reliability and equivalence of alternate forms for the Symbol Digit Modalities Test: implications for multiple sclerosis clinical trials. Mult Scler. 2012; 18: 1320-1325.

22) Benedict RH, DeLuca J, et al.: Validity of the Symbol Digit Modalities Test as a cognition performance outcome measure for multiple sclerosis. Mult Scler J. 2017; 23: 721-733.

23) McLean SG and Samorezov JE: Fatigue-induced ACL injury risk stems from a degradation in central control. Med Sci Sports Exerc. 2009; 41: 1661-1672.

24) Kipp K, Brown TN, et al: : Decision making and experience level influence frontal plane knee joint biomechanics during a cutting maneuver. J Appl Biomech. 2013; 29: 756-762.

25) Kadaba MP, Ramakrishnan HK, et al.: Measurement of lower extremity kinematics during level walking. J Orthop Res. 1990; 8: 383-392.

26) Davis RB, Õunpuu S, et al.: A gait analysis data collection and reduction technique. Hum Mov Sci. 1991; 10: 575-587.

27) Brown SR, Brughelli M, et al: Knee mechanics during planned and unplanned sidestepping: a systematic review and metaanalysis. Sports Med. 2014; 44: 1573-1588.

28) Besier TF, Lloyd DG, et al.: Muscle activation strategies at the knee during running and cutting maneuvers. Med Sci Sports Exerc. 2003; 35: 119-127.

29) Russell PJ, Croce RV, et al.: Knee-muscle activation during landings: developmental and gender comparisons. Med Sci Sports Exerc. 2007; 39: 159-170.

30) Iguchi $\mathrm{J}$, Tateuchi $\mathrm{H}$, et al.: The effect of sex and fatigue on lower limb kinematics, kinetics, and muscle activity during unanticipated side-step cutting. Knee Surg Sports Traumatol Arthrosc. 2014; 22: 41-48.

31) Padua D, Arnold BL, et al.: Fatigue, vertical leg stiffness, and stiffness control strategies in males and females. J Athl Train. 2006; 41: 294-304.

32) Boden BP, Dean GS, et al.: Mechanisms of anterior cruciate ligament injury. Orthopedics. 2000; 23: 573-578. 
33) Koga H, Nakamae A, et al:: Mechanisms for noncontact anterior cruciate ligament injuries: knee joint kinematics in 10 injury situations from female team handball and basketball. Am J Sports Med. 2010; 38: 2218-2225.

34) Krosshaug T, Nakamae A, et al.: Mechanisms of anterior cruciate ligament injury in basketball: video analysis of 39 cases. Am J Sports Med. 2007; 35: 359-367.

35) MacWilliams B, Wilson DR, et al.: Hamstrings cocontraction reduces internal rotation, anterior translation, and anterior cruciate ligament load in weight-bearing flexion. J Orthop Res. 1999; 17: $817-822$

36) Hewett TE, Ford KR, et al.: Understanding and preventing acl injuries: current biomechanical and epidemiologic considerations - update 2010. N Am J Sports Phys Ther. 2010; 5: 234251.

37) Sheridan LK, Fitzgerald HE, et al.: Normative Symbol Digit Modalities Test performance in a community-based sample. Arch Clin Neuropsychol. 2006; 21: 23-28.

38) Arendt E and Dick R: Knee injury patterns among men and women in collegiate basketball and soccer. NCAA data and review of literature. Am J Sports Med. 1995; 23: 694-701.

39) Agel J, Arendt E, et al.: Anterior cruciate ligament injury in na- tional collegiate athletic association basketball and soccer: a 13year review. Am J Sports Med. 2005; 33: 524-530.

40) Hewett TE, Torg JS, et al.: Video analysis of trunk and knee motion during non-contact anterior cruciate ligament injury in female athletes: lateral trunk and knee abduction motion are combined components of the injury mechanism. Br J Sports Med. 2009; 43: 417-422.

41) Sturnick DR, Van Gorder R, et al.: Tibial articular cartilage and meniscus geometries combine to influence female risk of anterior cruciate ligament injury. J Orthop Res. 2014; 32: 14871494.

42) Harmon KG and Dick R: The relationship of skill level to anterior cruciate ligament injury. Clin J Sport Med. 1998; 8: 260265.

43) Sigward S and Powers CM: The influence of experience on knee mechanics during side-step cutting in females. Clin Biomech. 2006; 21: 740-747.

44) Benedict RHB, Duquin JA, et al.: Repeated assessment of neuropsychological deficits in multiple sclerosis using the Symbol Digit Modalities Test and the MS Neuropsychological Screening Questionnaire. Mult Scler. 2008; 14: 940-946. 